\title{
Estilo de vida dos adolescentes e jovens adultos e comportamentos desviantes e delinquentes: Das vivências familiares, escolares e individuais
}

\section{Adolescents' and young adult's' lifestyle and deviant and delinquent behaviors: From family, school, and individual experiences}

\author{
Sónia Caridade (1) \\ Ana Cristina Martins (2) \\ Laura Nunes (1)
}

\begin{abstract}
(1) Faculdade de Ciências Humanas e Sociais, Centro de Investigação em Ciências Sociais e do Comportamento; Observatório Permanente Violência e Crime. Universidade Fernando Pessoa, Porto, Portugal.

(2) Faculdade de Ciências Humanas e Sociais, Universidade Fernando Pessoa, Porto, Portugal.
\end{abstract}

Recebido: 08/01/2019; Revisto: 13/02/2019; Aceite: 28/02/2019.

https://doi.org/10.31211/rpics.2019.5.1.106

\section{Resumo}

Objetivo: O conhecimento do estilo de vida dos jovens revela-se fundamental para a identificação e intervenção nos comportamentos de risco e na promoção de oportunidades de desenvolvimento dos jovens. Neste sentido, o presente trabalho tem por objetivo a caraterização do estilo de vida dos jovens e dos seus eventuais comportamentos desviantes e delinquentes. Método: A amostra foi constituída por 80 jovens $(M=19$ anos; $D P=2,60)$, sendo $56 \%$ rapazes. Para efeito da recolha de dados recorreu-se a um inquérito por questionário construído para o efeito do presente estudo. Resultados: Os inquiridos relataram inexistência de supervisão parental, falta de imposição de regras, existência de conflitos com os pares, professores e funcionários, bem como ausência de hábitos de estudo e de atividades extracurriculares; admitiram, ainda, passar mais tempo entre pares do que com os familiares. Admitiram, ainda, ter já adotado diferentes condutas desviantes e delinquentes ou mesmo criminais (e.g., agressões para com colegas, professores e funcionários, causar dano intencional em objetos de outros, estar envolvidos em grupos de pares desviantes, invadir propriedades privadas, e participar em furtos, e em tráfico de droga). Estes comportamentos foram mais assumidos por rapazes. Conclusões: Importa, deste modo, que os esforços de prevenção da delinquência considerem o grupo de jovens que precocemente manifestam comportamentos desviantes, dado o seu maior risco para o desenvolvimento de futuras formas de inadaptação social, incidindo igualmente sobre o meio escolar e familiar.

Palavras-Chave: Estilo de vida; Comportamentos delinquentes; Comportamentos desviantes; Adolescentes; Jovens adultos; Estudo de levantamento descritivo.

\section{DI\&D | ISMT}

rpics@ismt.pt

https://rpics.ismt.pt

\section{Publicação em Acesso Aberto}

(02019. O(s) Autor(es). Este é um artigo de acesso aberto distribuído sob a Licença Creative Commons Attribution, que permite uso, distribuição e reprodução sem restrições em qualquer meio, desde que o trabalho original seja devidamente citado.
Sónia Caridade

Faculdade de Ciências Humanas e Sociais da Universidade Fernando Pessoa, Praça 9 de Abril, 349, 424- 9004 Porto, Portugal e-mail:soniac@ufp.edu.pt 


\begin{abstract}
Objective: Knowledge about young people lifestyle is fundamental for identifying and intervening in risk behaviors. It is also important for promoting opportunities for young people's development. So, the present article aims to characterize adolescents and young adults' lifestyle and their possible delinquent behaviors. Method: The sample included 80 adolescents and young adults $(M=19.00, S D=2.60$ years old), $56 \%$ of them males. For the purpose of collecting data, we created a questionnaire for this study. Results: The participants reported lack of parental supervision, no rules enforcement; the occurrence of conflicts with peers, teachers and school staff, as well as absence of study habits and extracurricular activities; they also admitted to spending more time with peers than with family. They also admitted that they had already adopted different deviant and delinquent or even criminal conduct (e.g., assaults against colleagues, teachers and school staff, causing intentional damage to another person's objects, being involved with deviant peer groups, and having practices like the invasion of private property, thefts, and drug trafficking). The male sex assumed more of these behaviors. Conclusions: Therefore, in order to prevent crime, it is important to develop efforts to consider the group of adolescents and young adults who are already demonstrating antisocial behavior, given their greater risk for the development of future forms of social maladjustment, also affecting the school and family environment.
\end{abstract}

Keywords: Lifestyle; Delinquent behavior; Deviant behavior; Adolescents; Young adults; Descriptive survey study.

\title{
Introdução
}

A prática de condutas delinquentes por adolescentes e jovens tem sido alvo de inúmeras designações e conceptualizações consoante a perspetiva em análise (sociológica, legal/criminológica, psicopatológica/clínica) (Born, 2005; Negreiros, 2008). Neste trabalho adotaremos a conceptualização proposta pela abordagem sociológica dada a maior amplitude que confere ao conceito de delinquência, ao considerar que este pode abranger atos criminais e não criminais. A delinquência surge, assim, definida como integrando "comportamentos que se afastam significativamente de normas padrões de conduta e expectativas sociais estabelecidas numa determinada cultura" (Negreiros, 2016, p. 158). Não obstante esta ausência de consenso quanto ao conceito, tem sido amplamente defendido que a emergência deste tipo de práticas transgressivas em idades muito precoces encontra-se associada a um maior risco para o desenvolvimento de comportamentos delinquentes e criminais na adolescência e na vida adulta, respetivamente, em termos de severidade, frequência e duração (Negreiros, 2008).

A delinquência juvenil tem sido objeto de estudo desde há várias décadas, sendo extensa e sólida a investigação internacional nesta área (Braga \& Gonçalves, 2013). O estado da arte portuguesa sobre o fenómeno da delinquência não assume as mesmas proporções, mas já é notório o investimento científico, existindo um número considerável de estudos que procuram explorar diferentes aspetos do comportamento delinquente: i) caracterizar a extensão dos comportamentos delinquentes e identificar as características daqueles que os praticam (e.g., Braga \& Gonçalves, 2013; Carvalho, 2010; Gersão \& Lisboa, 1994; Mendes \& Carvalho, 2010); ii) analisar a relação entre certas dimensões do funcionamento (e.g., autoestima, narcisismo, traços psicopáticos, habilidades sociais) dos jovens com os comportamentos antissociais (e.g., Braga, Pechorro, Jesus, \& Gonçalves, 2018; Pechorro, Marôco, Vieira, Oliveira, \& Gonçalves, 2015; Sintra \& Formiga, 2012); iii) analisar como as variáveis da família e do grupo de pares se associam a diferentes percursos de reincidência (e.g., Cunha, Soares, Veríssimo, \& Matos, 2015) ou a 
influência do funcionamento familiar no autocontrolo (e.g., Gomes \& Gouveia-Pereira, 2014); iv) ou, ainda, os estudos que procuram dar voz aos/às jovens com percursos delinquentes na justiça de forma a melhor compreender as suas condutas delitivas (e.g., Conde \& Teixeira, 2018; Duarte, 2013; Duarte \& Carvalho, 2012; Matos, 2008).

Concomitantemente, e fruto desta vasta investigação, diversas abordagens teóricas emergiram, tais como as biológicas, psicológicas e sociais, no sentido de identificarem os principais fatores determinantes da delinquência (Nunes \& Trindade, 2015). De entre as diferentes abordagens existentes, destacamos os contributos da teoria do estilo de vida criminal de Walters (1990), a qual preconiza que os comportamentos e pensamentos associados a um padrão de vida criminal duradouro sofrem influência de três fatores: Condições, Escolhas e Cognições. A forma como as condições impulsionam o sujeito à adoção de condutas delinquentes resultam do tipo de experiências de vida nos primeiros anos, nomeadamente a forma como cada sujeito responde aos diferentes desafios que lhe são apresentados em termos de vinculação social, estimulação, busca de sensações e autoconceito (Walters, 1990). Estas condições, por sua vez, afetam as escolhas do sujeito, interagindo e influenciando-se mutuamente, contribuindo para a emergência do comportamento delitivo. As cognições, resultantes da união entre os outros dois fatores, são fundamentais na formação e manutenção do pensamento criminal e suportam o comportamento subsequente, alterando também o processo de tomada de decisão e a perceção sobre as variadas condições de vida (Walters, 1990).

O conhecimento do estilo de vida dos jovens e das suas rotinas diárias tem sido reconhecido como sendo fulcral na identificação e subsequente intervenção nos comportamentos de risco e no incremento de oportunidades de desenvolvimento dos jovens (Barbosa-Ducharne, Cruz, Marinho, \& Grande, 2012), fundamentando-se, deste modo, um maior investimento científico neste domínio. No presente trabalho, o conceito de estilo de vida é percebido como sendo multidimensional, englobando a interação de diferentes aspetos do funcionamento do jovem, incluindo as dinâmicas do seu funcionamento familiar, escolar, a forma como ocupa os seus tempos livres e o seu funcionamento individual (Barbosa-Ducharne et al., 2012). Numa outra perspetiva, também multidimensional, o estilo de vida surge percebido como sendo o resultado da combinação do tipo de lugares onde os jovens ocupam os seus tempos livres (e.g., locais públicos e semipúblicos, isentos de qualquer tipo de controlo social) das suas características, das pessoas com quem interagem nesses tempos livres (influência de pares) e do tipo de atividades desenvolvidas nesses momentos (tais como consumir álcool ou drogas ilegais) (Svensson \& Pauwels, 2010). Enquanto problema socialmente construído e parte integrante do processo de socialização dos jovens, a delinquência deverá ser compreendida em relação ao contexto em que é vivida, considerandose as experiências com os diversos agentes de socialização (e.g., a família, a escola, o grupo de pares e instituições de controlo formal (Carvalho, 2005).

A identificação dos fatores de risco constitui um aspeto fundamental na prevenção do comportamento delinquente. Apesar de alguns desses fatores serem comuns em muitos jovens com comportamentos delinquentes, a sua combinação poderá oscilar de indivíduo para indivíduo. A literatura tem vindo a identificar diferentes tipos de fatores de risco associados ao comportamento delinquente (Braga, Gonçalves, Basto-Pereira, \& Maia, 2017; Farrington, Gaffney, \& Ttofi, 2016; Forsyth et al., 2018), desde os fatores individuais/biológicos (e.g., complicações no parto, hiperatividade, temperamento), fatores familiares (e.g., comportamento antissocial dos pais, abuso de substâncias e precaridade e inconsistência 
da práticas parentais, maus tratos infantis), fatores relacionados com a escola e de forma mais concreta a associação a grupo de pares desviantes ou ainda fatores relacionados com a comunidade. Um estilo de vida de risco, que é assinalado pela presença de múltiplos de fatores de risco, incrementa a possibilidade de adoção de comportamentos antissociais e delinquentes (Svensson \& Pauwels, 2010).

A forma como os jovens escolhem/definem os seus hábitos de vida é, em grande medida, determinada pela influência exercida no contexto familiar e mais concretamente pelo apoio concedido pelos pais no decurso do seu desenvolvimento (e.g., Newman, Harrison, Dashiff, \& Davis, 2008; Walters, 2017), e sobretudo pelo tipo de práticas parentais adotadas. A este respeito cita-se a teoria geral do crime de Gottfredson e Hirschi (1990) que atribui a ausência de autocontrolo dos jovens às práticas parentais ineficazes e desadequadas, considerando o autocontrolo como sendo a principal causa do comportamento delinquente. Segundo esta abordagem teórica cabe aos pais criar e garantir as condições suscetíveis de promover as capacidades de autocontrolo das crianças e jovens, nomeadamente: i) estabelecer o vínculo dos pais à criança, suscetível de incrementar o seu desenvolvimento saudável; ii) assegurar a devida supervisão parental de forma a suprimir práticas desviantes; iii) monitorizar e identificar possíveis condutas desviantes; e, ainda, iv) corrigir o comportamento desviante, recorrendo-se a práticas educacionais equilibradas e proporcionais à conduta. Outros autores (e.g., Meldrum, Young, Hay, \& Flexon, 2012; Pauwels \& Svensson, 2009) têm reafirmado o importante papel que as práticas parentais eficazes e adequadas (i.e., pautadas por vinculação e supervisão parental) desempenham na promoção do autocontrolo dos jovens, dirimindo a possibilidade de adoção de comportamentos de risco e outras práticas transgressivas. De igual modo, o ajustamento do jovem em meio escolar e em termos psicossociais tem também sido associado ao modelo educacional que os pais adotam, e em que as manifestações de afeto, o tipo de comunicação familiar e as práticas disciplinares ajustadas constituem aspetos fulcrais no processo de formação e aprendizagem dos jovens (Newman et al., 2008). A exposição dos jovens a outras dinâmicas familiares, sobretudo a práticas abusivas e violentas (e.g., maus tratos infantis; exposição à violência doméstica; e.g., Braga et al., 2017; Sani, Nunes, \& Caridade, 2016) poderá também desempenhar um papel constrangedor no seu desenvolvimento, promovendo igualmente condutas não normativas. Considerando esta influência das vivências familiares ao nível da capacidade de autocontrolo e o comportamento delinquente dos jovens, Gomes e Gouveia-Pereira (2014) conduziram um estudo junto de 181 adolescentes, com idades compreendidas entre os 12 e os 19 anos de idade, inseridos em escolas da área da Grande Lisboa. Neste estudo, o funcionamento familiar revelou estar positivamente relacionado com o autocontrolo dos adolescentes, constituindo este um importante mediador da relação estabelecida entre o funcionamento familiar e a delinquência juvenil; além disso, os autores verificaram que quanto mais equilibrado for o sistema familiar (traduzido em níveis balanceados de coesão e flexibilidade familiar) maior a capacidade de autocontrolo dos adolescentes e a probabilidade de estes apresentarem um desenvolvimento individual saudável (Gomes \& Gouveia-Pereira, 2014). Por sua vez, a escola constitui um contexto privilegiado para a promoção e consolidação de múltiplas aprendizagens, propiciando também múltiplas interações (seja com pares, professores e outros agentes educativos) e condutas nem sempre favoráveis a um desenvolvimento normativo (e.g., Nunes, Caridade, \& Sani, 2017). De entre as condutas que se revelam mais problemáticas, destacam-se o uso de drogas 
(e.g., Nunes, Beça, \& Dinis, 2017), a prática de incivilidades, enquanto ações antissociais perturbadoras da harmonia social quotidiana (Garcia, 2006). Destaque, ainda, para a influência que as características do espaço físico escolar e sua configuração poderão ter no comportamento desviante dos jovens (Pimenta \& Nunes, 2017) ou mesmo a influência do grupo de pares na adoção de comportamentos delinquentes (Walters, 2016), e mais especificamente no consumo de drogas (Cardoso \& Malbergier, 2014).

A análise da delinquência juvenil em função da lente de género tem sido percebida como sendo marcadamente insuficiente e assinalada por uma perspetiva de género "por defeito", com a figura masculina tida como norma e omitindo-se a feminina (Duarte \& Carvalho, 2012, p. 3). Neste sentido, a variável género tem sido conceptualizada como elemento diferenciador do comportamento transgressivo e em que tradicionalmente se associa o sexo masculino à agressão e ao exercício da autoridade e o sexo feminino como sendo mais suscetível à vitimização (Chesney-Lind, 1997). Efetivamente, os estudos (e.g., Chesney-Lind, 1997; Duarte, 2013; Matos, 2008) conduzidos com raparigas em conflito com a lei sugerem a existência de algumas especificidades e diferenças ao nível do comportamento delinquente apresentado por rapazes e raparigas. Mais concretamente, têm sido identificadas especificidades sobretudo ao nível da trajetória transgressiva, dos fatores de risco e do impacto da conduta transgressiva (Hipwell \& Loeber, 2006). Neste sentido, tem sido defendido que na análise da delinquência juvenil no feminino, caracterizada por uma pluralidade de expressões e dimensões envolvidas, há que atender aos modos de vida das raparigas os quais parecem estar intrinsecamente associados a uma construção identitária de género (Duarte \& Carvalho, 2012).

Atendendo ao documentado impacto do estilo de vida dos jovens no seu comportamento desviante e/ou delinquente, o presente estudo tem como principal objetivo caracterizar o estilo de vida dos jovens, inseridos numa área rural, e o seu eventual comportamento desviante e/ou delinquente. De referir que grande parte dos estudos desenvolvidos sobre delinquência juvenil e comportamento desviante em Portugal foca-se nas realidades urbanas e em estilos de vida urbanos (e.g., Carvalho, 2010; Carvalho \& Duarte, 2013; Gomes \& Gouveia-Pereira, 2014), ou, ainda em jovens em conflito com a lei (Braga et al., 2018; Conde \& Teixeira, 2018; Cunha et al., 2015; Duarte, 2013) pelo que consideramos que o presente trabalho poderá constituir um importante contributo para o avanço do conhecimento neste domínio em Portugal. Mais especificamente, procurou-se: i) descrever o estilo de vida dos adolescentes e jovens, tomando em consideração as vivências familiares, escolares e individuais; ii) caracterizar os potenciais comportamentos desviantes e/ou delinquentes assumidos pelos adolescentes e jovens, nos últimos 12 meses anteriores à realização do estudo; iii) comparar a frequência dos comportamentos desviantes e/ou delinquentes em função do sexo dos participantes.

\section{Método}

\section{Participantes}

A amostra foi constituída por 80 adolescentes e jovens adultos, com idades entre os 15 e os 25 anos ( $M=$ 19 anos; $D P=2,60$ ). A escolha deste grupo etário deveu-se ao facto de este constituir um período particularmente propício ao envolvimento em comportamentos de risco e à adoção de condutas 
antissociais. Para além do critério idade, os adolescentes e jovens adultos teriam que residir em território nacional, do distrito de Viana do Castelo, encontrando-se inseridos em área rural. Não foram colocadas quaisquer outras restrições de participação, tendo a amostra sido constituída por adolescentes e jovens adultos que cumpriram os referidos critérios de inclusão e que aceitaram participar no presente estudo, consistindo numa amostra não probabilística, por conveniência.

Todos os participantes residiam no distrito de Viana do Castelo (norte de Portugal) e eram oriundos de uma área rural; 98\% tinham nacionalidade portuguesa e 3\% eram oriundos de outras nacionalidades (e.g., Cabo Verdiana). Quanto ao sexo, 56\% dos participantes pertenciam ao sexo masculino (56\%) e cerca de $43,8 \%$ ao sexo feminino. Relativamente ao ano escolar, cerca de $40 \%$ frequentava o ensino secundário, $40 \%$ o ensino superior e, em menor número, cursos profissionais (14\%) e o ensino básico (6\%).

No que ao agregado familiar diz respeito, a maioria dos inquiridos afirmou residir com os pais e os irmãos (61\%) ou apenas com os pais (20\%). Apenas 6\% relatou residir somente com a mãe e irmãos. Aproximadamente $25 \%$ dos participantes afirmou que não tinha irmãos; $35 \%$ indicou ter apenas um irmão; $28,7 \%$ dois irmãos e $11 \%$ três ou mais irmãos (Tabela 1).

\section{Tabela 1}

\section{Caracterização Sociodemográfica da Amostra}

\begin{tabular}{|c|c|c|c|}
\hline Variáveis & & Frequência Absoluta ( $n)$ & Frequência Relativa (\%) \\
\hline Residência & Viana do Castelo & 80 & 100 \\
\hline \multirow{2}{*}{ Nacionalidade } & Portuguesa & 78 & 98 \\
\hline & Outra & 2 & 3 \\
\hline \multirow{2}{*}{ Sexo } & Masculino & 45 & 56 \\
\hline & Feminino & 35 & 44 \\
\hline \multirow{4}{*}{ Ano Escolar } & Ensino básico & 5 & 6 \\
\hline & Ensino secundário & 32 & 40 \\
\hline & Ensino universitário & 32 & 40 \\
\hline & Ensino profissional & 11 & 13 \\
\hline \multirow{7}{*}{ Agregado Familiar } & Pai/mãe/irmãos & 49 & 61 \\
\hline & Pai/mãe & 16 & 20 \\
\hline & Mãe/irmãos & 5 & 6 \\
\hline & Só com a mãe & 3 & 4 \\
\hline & Pai/irmãos & 2 & 3 \\
\hline & Pais/irmãos/avós & 2 & 3 \\
\hline & Pais/avós/irmãos & 1 & 1 \\
\hline \multirow{4}{*}{ Número de Irmãos } & Sem irmãos & 20 & 25 \\
\hline & Um irmão & 28 & 35 \\
\hline & Dois irmãos & 23 & 29 \\
\hline & Três ou mais & 9 & 11 \\
\hline
\end{tabular}




\section{Materiais}

Para a recolha de dados recorremos a um inquérito por questionário desenvolvido para o efeito. Este encontrava-se estruturado em cinco grupos de questões: o primeiro grupo destinou-se a recolher a informação sociodemográfica dos adolescentes e jovens adultos (e.g., sexo, idade, grau de escolaridade, número de irmãos); o terceiro grupo de questões procurou explorar o funcionamento familiar dos participantes (e.g., identificar a profissão dos pais, caracterizar o ambiente e as dinâmicas familiares, a relação com os pais e irmãos), através de uma escala que vai de "Muito bom"; "Bom"; "Razoável"; a "Mau". Pretendeu também identificar-se as regras e rotinas familiares através de uma escala que começou em "Sempre", "Frequentemente", "Raramente" e "Nunca". Analisou-se seguidamente o funcionamento escolar/ocupacional dos adolescentes e jovens, designadamente a descrição do seu ambiente escolar, a sua com os professores, entre outros aspetos. Cada item deste grupo foi analisado através de uma escala que começou em "Muito bom", "Bom", "Razoável" e "Mau”. No quarto grupo, caracterizou-se o estilo de vida e a ocupação de tempos livres dos adolescentes e jovens adultos, pesquisando-se as atividades em que aqueles estavam envolvidos, através de uma escala que começou em "Todos os dias", "Uma vez por semana”, "Só aos fins de semana" e "Nunca", num conjunto de atividades desportivas, religiosas, de lazer, familiares e saídas noturnas. Por fim, no quinto grupo, caracterizou-se a frequência da execução de determinados comportamentos desviantes por parte dos adolescentes e jovens adultos, nos últimos 12 meses. Para esse fim, os participantes tiveram de identificar a frequência com que realizavam cada um dos comportamentos apresentados, considerando a seguinte escala: "Nunca", "Uma vez", "Duas ou três vezes", "Quatro vezes ou mais". Neste grupo de questões, foram listados, primeiramente, um conjunto de comportamentos desviantes (e.g., desrespeitar professores ou funcionários, faltar constantemente às aulas), seguindo-se os comportamentos delinquentes (e.g., prática de furtos, roubos, tráfico de drogas) de forma a identificar o tipo de comportamento em que o adolescente e jovem estavam inseridos.

\section{Procedimentos}

Para a realização do presente estudo tornou-se necessário proceder a pedidos de autorização formais junto das instituições envolvidas, tendo havido ainda submissão do projeto de investigação para validação pela comissão de ética da Universidade que acolheu a realização deste estudo em novembro de 2015.

Uma vez que o instrumento utilizado foi construído para o efeito do presente estudo, o mesmo foi submetido a um pré-teste junto de 10 jovens com as características da população a quem o questionário foi dirigido. O pré-teste foi acompanhado de reflexão falada, de forma a avaliar a eficácia e pertinência do questionário, considerando os seguintes aspetos: i) se as expressões usadas eram compreensíveis e inequívocas; ii) se a forma das questões usadas possibilitava coligir as informações pretendidas; iii) se o questionário não era muito extenso e não provocava desinteresse ou irritação; iv) e, ainda, se as questões não apresentavam ambiguidade.

A recolha de dados foi efetuada porta-a-porta (numa área geográfica delimitada e referenciada pelas autoridades locais como sendo problemática em matéria de delinquência juvenil) e individualmente, estando a investigadora presente no local de forma a esclarecer eventuais dúvidas em relação ao estudo. Após explicar o estudo, a investigadora procurou dar um espaço ao respondente para que este não se sentisse pressionado, 
promovendo um ambiente confortável e tranquilo que apenas era interrompido caso o participante assim o desejasse. Além disso, no sentido de assegurar os requisitos adequados para a administração do questionário e as condições de privacidade do respondente, foi-lhe dada a possibilidade de poder escolher um local em que se sentisse mais confortável e tranquilo para responder ao inquérito.

No caso dos menores de idade, solicitou-se, por escrito, a autorização e consentimento informado aos seus pais/tutores legais. A recolha do consentimento informado foi igualmente obtida junto dos que possuíam idade igual ou superior a 18 anos. Primeiramente, a investigadora informou os participantes e/ou pais/tutores legais sobre os objetivos do estudo, natureza anónima do mesmo, confidencialidade e uso meramente estatístico dos dados. Adicionalmente, adotou-se o particular cuidado em separar os formulários dos consentimentos informados assinados para impedir o seu emparelhamento e garantir o seu efetivo anonimato. Para tanto, a investigadora procedeu primeiramente à recolha dos consentimentos informados, colocando-os num envelope para esse efeito, e só posteriormente foram entregues os questionários.

Os dados recolhidos através do inquérito por questionário foram sujeitos a análises estatísticas com recurso ao programa informático IBM SPSS 23.0 (Statistical Package for the Social Sciences), tendo sido realizadas essencialmente análises descritivas para caracterização da amostra, do estilo de vida dos adolescentes e jovens segundo as vivências familiares, escolares e individuais, e dos comportamentos desviantes e/ou delinquentes dos adolescentes e jovens. Para analisar as diferenças de sexo em termos do envolvimento ou não envolvimento em comportamentos desviantes e/ou delinquentes foi realizado o teste $t$ de Student para amostras independentes (Marôco, 2010). Uma vez que o teste $t$ se mostrou parcialmente significativo foi calculado o tamanho de efeito (Espírito-Santo \& Daniel, 2015), o qual foi interpretado de acordo com as diretrizes de Cohen (1988). Para comparar as diferenças de sexo para os diferentes tipos de comportamentos desviantes e/ou delinquentes foi realizado o teste Mann-Whitney (Marôco, 2010). Neste caso, tratando-se de um teste não-paramétrico, procedeu-se ao cálculo do tamanho de efeito de acordo com as diretrizes de Rosenthal (1991), através da fórmula $r=\frac{z}{\sqrt{N}}$, em que $z=z$-score dado pelo SPSS e o $N$ é relativo aos participantes do estudo, e interpretado de acordo com os seguintes valores: $r=0,10$ indicador de um efeito pequeno; $r=0,30$ indicador de efeito médio e $r=0,50$ indicador de efeito amplo.

\section{Resultados}

\section{Estilo de vida dos adolescentes e jovens adultos segundo as vivências familiares, escolares e individuais}

Uma percentagem considerável (59\%) dos inquiridos qualificou o seu ambiente familiar como bom e 31\% de razoável. A relação com os pais foi classificada por $51 \%$ dos adolescentes e jovens como boa e por $24 \%$ de razoável; quanto à relação que os pais tinham entre si, $40 \%$ afirmou ser boa e 34\% de razoável; do mesmo modo a relação com os irmãos foi considerada pela maioria como boa (54\%) e razoável por $14 \%$. No que concerne às atividades em família, $43 \%$ classificou-as como sendo razoáveis e $36 \%$ como boas (Tabela 2). 
Tabela 2

Caracterização das Variáveis Relacionadas com o Funcionamento Familiar

\begin{tabular}{|c|c|c|c|c|c|c|c|c|}
\hline \multirow{2}{*}{$\begin{array}{l}\text { Variáveis relacionadas com o } \\
\text { funcionamento familiar }\end{array}$} & \multicolumn{2}{|c|}{ Muito bom } & \multicolumn{2}{|c|}{ Bom } & \multicolumn{2}{|c|}{ Razoável } & \multicolumn{2}{|c|}{ Mau } \\
\hline & $n$ & $\%$ & $n$ & $\%$ & $n$ & $\%$ & $n$ & $\%$ \\
\hline Ambiente familiar & 6 & 8 & 47 & 59 & 25 & 31 & 2 & 3 \\
\hline Relação com os pais & 19 & 24 & 41 & 51 & 19 & 24 & 1 & 1 \\
\hline Relação entre os pais & 10 & 13 & 32 & 40 & 27 & 34 & 6 & 8 \\
\hline Relação com os irmãos & 11 & 14 & 43 & 54 & 11 & 14 & - & - \\
\hline Atividades em família & 4 & 5 & 29 & 36 & 34 & 43 & 13 & 16 \\
\hline
\end{tabular}

A imposição de regras no dia-a-dia foi admitida por $51 \%$, sendo que $23 \%$ referiu que os pais raramente estabeleciam regras. Percentagens similares foram apuradas para a existência de imposição de tarefas e obrigações no dia-a-dia, admitidas frequentemente por $49 \%$ dos participantes ao contrário de $28 \%$ que afirmou raramente ter tarefas a cumprir. Relativamente à imposição de horários de chegada a casa em saídas noturnas, $44 \%$ mencionou que raramente tinha horários para chegar a casa e $38 \%$ que frequentemente tinha de cumprir horários. Do mesmo modo, $45 \%$ respondeu que era raro ter castigos em caso de incumprimento das regras ao contrário de 35\% dos participantes. Também $46 \%$ admitiu que raramente eram definidos horários de estudo pelos pais e $26 \%$ mencionou que tal ocorria frequentemente. Um número expressivo de adolescentes e jovens (46\%) referiu que os pais incentivavam à prática de atividades extracurriculares de forma frequente, contudo uma percentagem significativa (33\%) afirmou tal não acontecer. Por outro lado, $50 \%$ dos adolescentes e jovens adultos referiu, ainda, que tinha de informar os pais sobre quem constituía o seu grupo de pares, ao contrário de $26 \%$ que admitiu não ter de o fazer. Do mesmo modo, $48 \%$ mencionou informar os pais acerca dos locais que frequentava de forma frequente e $28 \%$ respondeu que era raro fazê-lo. Já quanto às atividades noturnas realizadas com os pares no $41 \%$ respondeu que era raro partilhar esta informação com os pais ao contrário do manifestado por $35 \%$ (Tabela 3).

\section{Tabela 3}

Caracterização das Variáveis Relacionadas com o Funcionamento Familiar (cont.)

\begin{tabular}{lcccccccc}
\hline \multirow{2}{*}{$\begin{array}{l}\text { Variáveis relacionadas com o funcionamento } \\
\text { familiar (cont.) }\end{array}$} & \multicolumn{2}{c}{ Sempre } & \multicolumn{2}{c}{ Frequentemente } & \multicolumn{2}{c}{ Raramente } & Nunca \\
\cline { 2 - 10 } & $n$ & $\%$ & $n$ & $\%$ & $n$ & $\%$ & $n$ & $\%$ \\
\hline Imposição de regras & 13 & 16 & 41 & 51 & 19 & 23 & 7 & 9 \\
\hline Imposição de tarefas e obrigações & 15 & 19 & 39 & 49 & 22 & 28 & 4 & 5 \\
\hline Imposição de horários de chegada casa & 8 & 10 & 30 & 38 & 35 & 44 & 7 & 9 \\
\hline Castigos pelo incumprimento de regras & 4 & 5 & 28 & 35 & 36 & 45 & 12 & 15 \\
\hline Definição de horários para estudo & 6 & 8 & 21 & 26 & 37 & 46 & 15 & 19 \\
\hline Incentivo atividades extracurriculares & 6 & 8 & 37 & 46 & 26 & 33 & 10 & 13 \\
\hline Informar os pais sobre o grupo de pares & 14 & 18 & 40 & 50 & 21 & 26 & 5 & 6 \\
\hline $\begin{array}{l}\text { Informar os pais sobre os locais que } \\
\text { frequentam à noite }\end{array}$ & 16 & 20 & \multirow{2}{*}{38} & 48 & 22 & 28 & 4 & 5 \\
\hline $\begin{array}{l}\text { Informar os pais sobre as atividades que fazem } \\
\text { com os amigos em saídas noturnas }\end{array}$ & 14 & 18 & 28 & 35 & 33 & 41 & 5 & 6 \\
\hline
\end{tabular}


No que respeita ao funcionamento escolar, $65 \%$ dos participantes admitiu nunca ter reprovado de ano e uma amostra expressiva (33\%) admitiu retenções de ano, sendo que $26 \%$ referiu que tal já aconteceu pelo menos uma vez.

\section{Tabela 4}

Caraterização das Variáveis Associadas ao Funcionamento Escolar

\begin{tabular}{|c|c|c|c|c|c|c|c|c|}
\hline \multirow[b]{2}{*}{ Variáveis associados ao funcionamento escolar } & \multicolumn{2}{|c|}{ Muito bom } & \multicolumn{2}{|c|}{ Bom } & \multicolumn{2}{|c|}{ Razoável } & \multicolumn{2}{|c|}{ Mau } \\
\hline & $n$ & $\%$ & $n$ & $\%$ & $n$ & $\%$ & $n$ & $\%$ \\
\hline Aproveitamento escolar & 2 & 3 & 31 & 39 & 35 & 44 & 12 & 15 \\
\hline Assiduidade & 4 & 5 & 34 & 43 & 30 & 38 & 12 & 15 \\
\hline Participação ativa nas aulas & 29 & 36 & 35 & 44 & 16 & 20 & - & - \\
\hline Hábitos de estudo & 1 & 1 & 27 & 34 & 32 & 40 & 20 & 25 \\
\hline Ambiente escolar & 6 & 8 & 46 & 58 & 25 & 31 & 3 & 4 \\
\hline Relação com professores & 3 & 4 & 44 & 55 & 28 & 35 & 5 & 6 \\
\hline Relação com funcionários & 2 & 3 & 43 & 54 & 29 & 36 & 6 & 8 \\
\hline Relação com colegas & 10 & 13 & 50 & 63 & 20 & 25 & - & - \\
\hline
\end{tabular}

Ainda que $39 \%$ classificasse o seu aproveitamento escolar como bom, a maioria (44\%) considerou ser razoável, e 15\% classificou-o como mau. Em termos de assiduidade, 43\% admitiu possuir uma boa assiduidade, 38\% referiu ser razoável e 15\% reconheceu possuir uma má assiduidade. Da mesma forma, 44\% afirmou ter boa participação nas aulas, 36\% apontou uma participação muito boa e $20 \%$ classificou-a como razoável. Os hábitos de estudo foram classificados por $40 \%$ como sendo razoáveis, por 34\% como bons e $25 \%$ admitiu possuir maus hábitos de estudo. O ambiente escolar foi qualificado pela maioria (58\%) como sendo bom e por $31 \%$ de razoável. De igual modo, a maioria dos participantes (55\%) descreveu a relação com os professores como sendo boa e 35\% de razoável, o mesmo se verificando em relação aos funcionários (de $54 \%$ classificou a relação com sendo boa e $36 \%$ apontou como sendo razoável) e aos colegas, tendo a maior parte respondido que possuía uma boa relação com estes (63\%) e $25 \%$ classificou a relação como sendo razoável (Tabela 4).

Dos que admitiram a prática de atividades extracurriculares (54\%), 35\% referiu o envolvimento em atividades desportivas (e.g., ginásio, futebol). Por fim, 36\% admitiu que já se tinha envolvido em algum tipo de problema na escola, com 31\% a admitir a implicação em agressões físicas, verbais ou psicológicas com os colegas.

Interpelados sobre com quem e como ocupam os tempos livres, a grande maioria (69\%) dos participantes respondeu fazê-lo com amigos ou namorada/o e apenas $20 \%$ afirmou permanecer sozinhos/as ou com os pais e avós. Quanto ao local escolhido para tal, 70\% identificou o café ou escola e 31\% a casa. 
Tabela 5

Caracterização das Atividades Realizadas nos Tempos Livres

\begin{tabular}{|c|c|c|c|c|c|c|c|c|c|c|}
\hline \multirow[b]{2}{*}{ Atividades realizadas nos tempos livres } & \multicolumn{2}{|c|}{$\begin{array}{c}\text { Todos os } \\
\text { dias }\end{array}$} & \multicolumn{2}{|c|}{$\begin{array}{c}>1 \text { vez por } \\
\text { semana }\end{array}$} & \multicolumn{2}{|c|}{$\begin{array}{l}1 \text { vez por } \\
\text { semana }\end{array}$} & \multicolumn{2}{|c|}{$\begin{array}{c}\text { Aos fins-de- } \\
\text { semana }\end{array}$} & \multicolumn{2}{|c|}{ Nunca } \\
\hline & $n$ & $\%$ & $n$ & $\%$ & $n$ & $\%$ & $n$ & $\%$ & $n$ & $\%$ \\
\hline Atividades desportivas & 1 & 1 & 15 & 19 & 7 & 9 & 39 & 49 & 18 & 23 \\
\hline Realizar tarefas em conjunto com a família & 1 & 1 & 16 & 20 & 17 & 22 & 22 & 28 & 24 & 30 \\
\hline Navegar na internet & 55 & 69 & 24 & 30 & 1 & 1 & - & - & - & - \\
\hline Frequentar redes sociais & 39 & 49 & 32 & 40 & 7 & 9 & - & - & 2 & 3 \\
\hline Ler e escrever & 5 & 6 & 23 & 29 & 15 & 19 & 7 & 8 & 31 & 39 \\
\hline Ver televisão & 44 & 55 & 35 & 44 & 1 & 1 & - & - & - & - \\
\hline Ir ao cinema & - & - & 4 & 5 & 5 & 6 & 47 & 59 & 24 & 30 \\
\hline Jogar jogos & 22 & 28 & 18 & 23 & 2 & 3 & 8 & 10 & 30 & 38 \\
\hline
\end{tabular}

Quanto ao tipo de atividades realizadas nos tempos livres, $49 \%$ admitiu a prática de atividades desportivas sobretudo ao fim-de-semana, período este igualmente destinado para a realização de tarefas em conjunto com a família (e.g., caminhadas, passeios ao ar livre), segundo $28 \%$ dos participantes, tendo $30 \%$ afirmado nunca realizar tarefas em conjunto com a família. Como atividades de lazer individuais, a maioria (69\%) assumiu navegar na internet todos os dias e $30 \%$ mais do que uma vez por semana; $49 \%$ afirmou frequentar as redes sociais diariamente e $40 \%$ mais do que uma vez por semana; $39 \%$ afirmou que nunca lia ou escrevia e 19\% referiu fazê-lo apenas uma vez por semana; 55\% revelou ter por hábito ver televisão todos os dias e 44\% mais do que uma vez por semana; $59 \%$ admitiu ir ao cinema só aos fins-de-semana e 30\% nunca ter ido; $28 \%$ admitiu jogar jogos de vídeo todos os dias; $23 \%$ mais do que uma vez por semana (Tabela 5).

Quanto às saídas noturnas, $70 \%$ respondeu que tinha por hábito sair com os amigos e $25 \%$ com os amigos e com a namorada. Como atividades realizadas nas saídas à noite, $59 \%$ identificou atividades em grupos de pares (e.g., beber, fumar, dançar, conversar) e $41 \%$ respondeu que ia sempre e frequentemente ter com a namorada/o; como locais frequentados $68 \%$ respondeu que frequentemente tinham por hábito ir a bares, discotecas e pubsou locais de diversão noturna.

Interpelados sobre eventuais experiências relacionadas com consumos de substâncias, $55 \%$ respondeu que consumiu tabaco. Destes, $40 \%$ admitiu que experimentou tabaco entre os $15-18$ anos e $32 \%$ revelou ter experimentado em idades precoces como os 10-14 anos. Quanto ao consumo de bebidas alcoólicas, cerca de $66 \%$ referiu que apenas bebia ao fim-de-semana, sendo que cerca de $43 \%$ experimentou beber entre os 15-18 anos e 38\% admitiu beber entre os 10-14 anos. Quanto ao consumo de outras drogas, cerca de $29 \%$ respondeu que consumia mais do que uma vez por semana; $16 \%$ todos os dias e $10 \%$ apenas ao fim-desemana. Quanto ao tipo de drogas consumidas: 36\% afirmou consumir "erva" ou "cannabis"; $18 \%$ "pólen" e 9\% "haxixe". A maior parte (81\%) dos participantes, que assumiu algum tipo de consumo, admitiu igualmente ter experienciado alterações comportamentais, tais como: "não estão no seu estado normal e ficam sem consciência dos seus atos" (54\%); "altera-Ihes o sistema nervoso podendo alguns ficar mais agressivos e outros mais relaxados, depende para o que Ihes dá" (16\%); 15\% respondeu não soube explicar as aludidas alterações. Quanto aos locais escolhidos para os consumos, $62 \%$ nomeou espaços de convívio 
como o café, ou então, em locais de diversão noturna como bares e discotecas (32\%) e $25 \%$ no café ou em casa quando tivessem sozinhos, ou na presença de amigos.

\section{Caracterização dos comportamentos desviantes e/ou delinquentes dos adolescentes e jovens adultos}

No que respeita aos comportamentos desviantes que os jovens assumiram praticar, pelo menos uma vez nos últimos 12 meses anteriores ao inquérito, verificamos que os mais reportados foram: envolvimento num grupo de amigos que se reúnem p/ameaçar, agredir os outros (74\%); envolvimento em lutas/pancadaria na escola, casa ou rua (68\%); o desrespeito ou até agressões a professores ou funcionários (66\%) e as faltas às aulas ou até mesmo reprovações (49\%) (Tabela 2). De entre os comportamentos delinquentes mais assumidos pelos adolescentes e jovens adultos destacam-se: danificar intencionalmente objetos de outrem (61\%); a entrada em propriedades privadas (58\%); prática de furtos (45\%); o envolvimento em tráfico de drogas (33\%) (Tabela 6).

\section{Tabela 6}

Comportamentos Desviantes e/ou Delinquentes Relatados pelos Participantes

\begin{tabular}{|c|c|c|c|c|}
\hline \multirow[b]{2}{*}{ Comportamentos } & \multicolumn{2}{|c|}{ Nunca } & \multicolumn{2}{|c|}{ Pelo menos 1 vez } \\
\hline & $n$ & $\%$ & $n$ & $\%$ \\
\hline Faltar ao respeito ou até agredir professores e funcionários & 21 & 26 & 46 & 66 \\
\hline Faltar constantemente às aulas, reprovar por faltas & 41 & 51 & 39 & 49 \\
\hline Fugas de casa & 79 & 99 & - & - \\
\hline Ser suspenso ou expulso da escola & 73 & 92 & 7 & 9 \\
\hline Danificar intencionalmente objetos de outrem & 32 & 40 & 48 & 61 \\
\hline Envolvimento em lutas/pancadaria na escola, casa ou rua & 26 & 33 & 54 & 68 \\
\hline Forçar a entrada em propriedades privadas & 33 & 41 & 46 & 58 \\
\hline Envolvimento com amigos para ameaçar, agredir os outros & 21 & 26 & 59 & 74 \\
\hline Venda de material roubado & 67 & 84 & 13 & 16 \\
\hline Uso de armas ilegais & 78 & 98 & 2 & 3 \\
\hline Venda/Tráfico de drogas & 51 & 64 & 26 & 33 \\
\hline Prática de furtos & 44 & 55 & 36 & 45 \\
\hline
\end{tabular}

\section{Comportamentos desviantes e/ou delinquentes dos adolescentes e jovens adultos em função do sexo}

Analisando os comportamentos desviantes e/ou delinquentes admitidos pelos participantes em função do sexo, encontramos diferenças de sexo parcialmente significativas, e em que os rapazes admitiram mais ( $M$ $=21,8 ; D P=5,05)$ comparativamente com as raparigas $(M=18,9 ; D P=3,35)$ o recurso a algum tipo de comportamento desviante e/ou delinquente $[t(78)=2,842 ; p=0,006 ; / C 95 \%(0,18-1,09) ; d$ de Cohen $=$ $0,66]$. Mais concretamente, foram detetadas diferenças de sexo estatisticamente significativas nos seguintes comportamentos: danificar intencionalmente objetos de alguém $(U=610,50 ; Z=-1,988 ; p=$ 0,047; $r=-0,22)$; venda de algum tipo de material roubado $(U=599,50 ; Z=-2,850 ; p=0,007 ; r=-0,32)$; tráfico/venda de drogas $(U=546,00 ; Z=-2,751 ; p=0,006 ; r=-0,31)$; prática de algum tipo de furto $(U=$ 
$613,50 ; Z=-1,924 ; p=0,054 ; r=-0,21)$; prática de algum tipo de roubo (e.g., assaltos a lojas, casas, carteiras) $(U=682,50 ; Z=-2,231 ; p=0,026 ; r=-0,25)$ (Tabela 7$)$.

Tabela 7

Comportamentos Desviantes e/ou Delinquentes Admitidos por Rapazes e Raparigas

\begin{tabular}{llcc}
\hline Comportamentos Desviantes e/ou Delinquentes & $\begin{array}{c}\text { Rapazes }(n=45) \\
\text { Média }\end{array}$ & $\begin{array}{c}\text { Raparigas }(n=35) \\
\text { Média }\end{array}$ & \multicolumn{2}{c}{$\boldsymbol{Z}$} \\
\hline Faltar ao respeito ou agredir professores e funcionários & 43,11 & 37,14 & $-1,273$ \\
\hline Faltar constantemente às aulas, reprovar por faltas & 42,07 & 38,49 & $-0,761$ \\
\hline Fugas de casa & 40,89 & 40,00 & $-0,882$ \\
\hline Ser suspenso ou expulso da escola & 42,33 & 38,14 & $-1,635$ \\
\hline Danificar intencionalmente objetos de alguém & 44,43 & 35,44 & $-1,988^{*}$ \\
\hline Envolvimento em lutas na rua, escola, casa & 43,43 & 36,73 & $-1,401$ \\
\hline Forçar entrada em propriedades privadas & 42,38 & 38,09 & $-0,892$ \\
\hline Envolvimento com amigos para ameaçar, agredir os outros & 43,89 & 36,14 & $-1,584$ \\
\hline Venda de algum tipo e material roubado & 44,68 & 35,13 & $-2,850^{*}$ \\
\hline Uso de armas ilegais & 41,28 & 39,50 & $-1,255$ \\
\hline Tráfico/venda de drogas & 45,87 & 33,60 & $-2,751^{*}$ \\
\hline Prática de algum tipo de furto & 44,37 & 35,53 & $-1,92^{+}$ \\
\hline Prática de algum tipo de roubo & 42,83 & 37,50 & $-2,231^{*}$ \\
\hline
\end{tabular}

Nota. $U=$ teste de Mann-Whitney; $Z=Z$-score; $r=$ estimativa do tamanho do efeito.

${ }^{*} p<0,05$ - nível de significância.

+ marginalmente significativo.

\section{Discussão}

A caracterização do estilo de vida dos jovens, tomando em consideração as vivências familiares, escolares e individuais, revela-se crucial para melhor compreender o comportamento dos mesmos, seja em termos prossociais ou antissociais e a partir de então delinear políticas de promoção e prevenção, respetivamente, destas condutas.

No presente estudo, e no que concerne ao ambiente familiar e às dinâmicas inerentes a este contexto de vida, uma grande parte dos jovens (59\%) avaliou positivamente o seu ambiente familiar (e $31 \%$ como razoável), situando a realização de atividades em família sobretudo no período de fim-de-semana, algo que poderá ser explicado pelo facto de uma percentagem considerável de participantes se encontrar a frequentar o ensino superior (40\%). Além disso, é comum na faixa etária em que se encontram os participantes (15-25), e de forma mais particular no período da adolescência, se verificar um afastamento em relação aos pais, motivado pelo facto do quotidiano destes jovens estruturar-se fundamentalmente em torno do grupo de pares, tal como apurado por Cunha et al. (2015). Além disso e como bem lembram Gomes e Gouveia-Pereira (2014), embora os jovens possam passar algum tempo separados das famílias, o importante é que a tomada de decisões seja partilhada, haja um tipo de liderança democrática, existem papéis estáveis que possam ser partilhados e que as regras familiares se revelem consistentes e consentâneas com as necessidades desenvolvimentais dos seus elementos. 
Ainda no que respeita às dinâmicas familiares, uma percentagem considerável apontou como sendo raro as seguintes imposições e/ou obrigações: presença de regras (23\%), tarefas e obrigações (28\%), horários de entrada em casa à noite (44\%), horários para estudar (46\%), castigos perante o incumprimento das regras (45\%) ou mesmo o incentivo à realização de atividades extracurriculares (33\%). Tais dados parecem fazer sobressair uma certa precariedade da supervisão parental e das práticas educativas adotadas, algo que poderá estar relacionado com a diminuída capacidade que os pais possuem nestas idades (especialmente na adolescência) devido à maior influência do grupo de pares, para impor disciplina e exercer o devido controlo social (Içli \& Çoban, 2012). Contudo, não será de negligenciar as evidências que comprovam que a falta de supervisão parental, a ausência de regras e hábitos de disciplina a par da reduzida disponibilidade de tempo que os pais dedicam às tarefas em conjunto com os filhos e ao facto de as crianças e/ou jovens passaram a maior parte do seu tempo em contexto escolar, encontram-se associados ao maior risco para os jovens adotarem condutas desviantes em virtude da ausência de limites convencionais e do afastamento do vínculo afetivo (Bidarra, Vaz-Rebelo, Barreira, Alferes, \& Pereira, 2017). Efetivamente, a investigação tem vindo a documentar a importância dos estilos educativos parentais e da qualidade da vinculação na regulação e gestão de certos comportamentos dos adolescentes. A título exemplificativo, Simões, Ferreira, Braga e Vicente (2015) verificaram que o comportamento parental que se baseia em suporte afetivo constitui um fator protetivo importante na adoção de certos comportamentos específicos, como é o caso do bullying. Mais especificamente, neste estudo, a agressividade surgiu sobretudo associada à rejeição e reduzido suporte emocional, bem como a uma menor comunicação e confiança em relação à figura paterna (Simões et al., 2015). Além disso, sabe-se, ainda, que o controlo e apoio parental ajudam a moldar a autoeficácia dos jovens na adoção de um estilo de vida convencional, e esta, por sua vez, reduz a probabilidade de aqueles se envolverem em comportamento delinquentes futuros (Walters, 1990, 2017). $\mathrm{Na}$ análise do funcionamento escolar dos participantes, foi possível apurar a existência de um número expressivo de retenções escolares. Ademais, os participantes qualificaram o seu aproveitamento escolar como razoável, admitindo ausência de hábitos de estudos; uma percentagem significativa de estudantes (31\%) avaliou o seu ambiente escolar como razoável, qualificando de igual forma (como razoável) a relação com os seus professores e funcionários. Tais dados afiguram-se preocupantes se considerarmos que: i) o sucesso académico ou o alcance de determinadas metas tende a influenciar a performance dos comportamentos prossociais dos jovens (Almeida-Paiva \& Lourenço, 2010); e ii) além disso, um bom ambiente escolar tende a fomentar relações interpessoais saudáveis entre alunos, professores e funcionários como também previne comportamentos violentos entre os jovens (Leão, 2010): iii) a ausência de experiências escolares positivas e de sucesso poderá constituir um facilitador da desintegração escolar e consequentemente conduzir ao envolvimento em condutas desviantes, tal como o apurado no estudo de Conde e Teixeira (2018) numa amostra de jovens portugueses em conflito com a lei. Um número considerável de participantes (36\%) admitiu, ainda, o envolvimento em agressões físicas, verbais ou psicológicas com os colegas, corroborando o evidenciado por outros estudos (e.g., Cho, Wooldredge, \& Park, 2016; Simões et al., 2015) de que o bullyingé uma realidade muito frequente em meio escolar e com repercussões futuras, podendo contribuir para um maior risco de perpetuação dos comportamentos violentos na idade adulta (Leão, 2010).

Em termos de funcionamento individual e embora os participantes tenham verbalizado um aumento crescente da prática de exercício físico (idas ao ginásio), comparativamente com as outras atividades 
listadas no instrumento (e.g., atividades religiosas), uma percentagem considerável dos jovens inquiridos revelou possuir hábitos de vida mais sedentários (e.g., 68\% afirmou não praticar qualquer atividade física). Como atividades de lazer preferenciais, os participantes identificaram como sendo mais frequente (todos os dias) navegar na internet, nas redes sociais e visualização de programas televisivos, sendo que outras atividades como ler ou escrever eram menos frequentes. Efetivamente, tem sido documentado um aumento expressivo das práticas digitais e do funcionamento em rede, sobretudo por parte dos jovens (Amaral, Reis, Lopes, \& Quintas, 2017; Instituto Nacional de Estatística, 2017), motivado pelos crescimento e avanço tecnológicos registados nas últimas décadas. Um estudo conduzido por Amaral et al. (2017) ao nível nacional entre 2014 e 2015, em 18 capitais de distrito de Portugal Continental, com estudantes de diferentes níveis de ensino (básico, secundário e profissional) comprovou a relação intensa da rede com as práticas juvenis quotidianas, pela frequência diária de utilização e pelo tempo médio de exposição, elucidando desde modo a transformação introduzida pelos media na forma como os jovens comunicam e interagem entre si; além disso, este estudo registou uma preponderância das práticas mais lúdicas (apuradas pelos elevados índices de consumos de práticas de entretenimento), e das ações e interações que possibilitem obter recursos para operar juntos dos seus pares, sendo escassa a procura de informação que possa apoiar o desempenho escolar. Se é certo que o recurso às ferramentas digitais poderá acarretar benefícios para o processo de socialização dos jovens (e.g., capacidade para exercitar o autocontrolo, promover a tolerância e respeito pelo outro, exercitar pensamento crítico) poderá também despoletar a emergência de outras situações de risco (Pujazon-Zazik \& Park, 2010), na medida em que tornam os jovens mais acessíveis e vulneráveis a maior intrusividade interpessoal.

Os locais de diversão noturna (e.g., bares, discotecas e pubs) foram identificados como os preferidos e mais frequentados pelos participantes, bem como os locais onde habitualmente ocorrem os consumos de álcool ou drogas (e.g., haxixe, cannabis), admitidos pelos participantes nos últimos 12 meses, podendo ocorrer também em casa e em cafés. Efetivamente, os estudos (e.g., Malta et al., 2011) comprovam que o uso de álcool e drogas na adolescência (a experimentação de bebidas alcoólicas em idades precoces foi de 71,4\% e o consumo regular de álcool rondava os $27,3 \%$ ) são muito frequentes e que o risco de consumo aumenta com as facilidades que eles tem em adquirir estas substâncias lícitas e ilícitas em locais de diversão noturna (e.g., festas, bares, discotecas e lojas de consumos ou até mesmo nas suas próprias casas). Os consumos assumidos pelos participantes do nosso estudo ocorreram em idades bastante precoces, entre os 10-14 anos e os 15-18 anos, algo que corrobora o encontrado em outros trabalhos anteriores (e.g., Batista, Carvalho, Pontes, \& Antunes, 2015).

Um outro objetivo do presente estudo envolvia a análise dos eventuais comportamentos desviantes ou delinquentes adotados pelos jovens nos últimos 12 meses, tendo os jovens admitido sobretudo o seu maior envolvimento em condutas antissociais tais como: desrespeito ou agressões a professores e funcionários (58\%), o dano intencional de objetos de outra pessoa (58\%) e o envolvimento em lutas, brigas ou pancadaria (33\%), sendo que uma grande parte admitiu que já o tinha feito pelo menos uma vez. Esta maior assunção deste tipo de comportamentos poderá ser explicada pelo facto de os jovens, neste tipo de inquéritos, assumirem sobretudo a prática de atos de menor gravidade (Dias, 2016). Também no estudo conduzido por Dalosto e Alencar (2013) os jovens assumiram adotar, em meio escolar, sobretudo as agressões físicas (e.g., socos, pontapés, empurrões) e as agressões psicológicas (e.g., humilhações, exclusão do grupo e danificar ou atirar objetos de outros intencionalmente). 
Um outro resultado algo preocupante apurado no presente estudo refere-se ao facto de os participantes terem admitido o seu envolvimento (uma ou mais vezes) em grupos de pares com comportamentos antissociais (e.g., reunirem de forma a ameaçar ou agredir os outros). Efetivamente a relação entre associação a grupos de pares delinquentes e o desenvolvimento de comportamento delinquente futuro tem sido bastante documentada, existindo vários esforços no sentido de identificar os mecanismos que promovem esta relação (e.g., desde modelagem, transferência de atitudes, atividades rotineiras, pensamento criminal proativo) (Walters, 2016).

Por fim, foram detetadas diferenças de sexo parcialmente significativas ao nível dos comportamentos desviantes/e ou delinquentes dos participantes e em que os rapazes assumiram mais, em comparação com as raparigas adotar algum tipo de comportamento desviante e/ou delinquente, tais como: danificar intencionalmente objetos de alguém; venda de algum tipo e material roubado; tráfico/venda de drogas; prática de algum tipo de furto; prática de algum tipo de roubo (e.g., assaltos a lojas, casas, carteiras). Este dado é de resto congruente com o apurado em outros estudos nacionais (e.g., Braga \& Gonçalves, 2013; Fonseca, 2013), onde o comportamento antissocial surge como sendo mais frequente nos rapazes do que nas raparigas.

\section{Conclusão}

A literatura da especialidade comprova que a adoção de comportamentos desviantes e/ou delinquentes é uma realidade presente em idades cada vez mais precoces. Este disfuncionamento comportamental está, não raras vezes, associado ao estilo de vida dos jovens, às dinâmicas familiares, e aos aspetos individual, escolar e social. Efetivamente, no presente estudo identificou-se uma percentagem expressiva de jovens que assumiram consumos diversos (e.g., tabaco, álcool e cannabis) muito precocemente, o que parecer coexistir com certas práticas parentais menos adequadas e funcionais, como reduzida supervisão e controlo parentais. Foi igualmente possível constatar o envolvimento de jovens em grupos de pares desviantes, seja em meio escolar, seja nas saídas noturnas. Acrescem ainda evidências que apontam para um cada vez maior envolvimento dos jovens ao nível das redes sociais em detrimento de outras atividades extracurriculares, desportivas e de lazer, bem como o seu envolvimento em comportamentos desviantes e/ou delinquentes, praticados sobretudo nos espaços escolar e de convívio social juvenil, considerados de risco para possíveis consumos de drogas. $O$ facto de a amostra dos participantes no estudo ser predominantemente oriunda de áreas rurais, constitui a nosso ver um aspeto inovador no presente estudo, atendendo a que grande parte dos estudos desenvolvidos sobre delinquência juvenil e comportamento desviante focam-se nas realidades urbanas e em estilos de vida urbanos.

A realização deste estudo não está, contudo, isenta de limitações que poderão ter influenciado os resultados e impedido de alcançar uma leitura mais holística do fenómeno. Desde logo trata-se de um estudo exploratório, conduzido numa determinada localidade do país (zona norte) e, como tal, não é possível a generalização dos resultados. Além disso, trata-se de uma amostra de conveniência, modesta, pelo que seria importante que em estudos futuros se contemplasse uma amostra mais alargada; acresce, ainda, o facto de se tratar de um estudo fundamentalmente descritivo, fundamentando-se a necessidade de se desenvolverem estudos correlacionais e até mesmo longitudinais, que possibilitassem compreender a evolução dos comportamentos transgressivos ao longo do tempo. 
Face aos dados obtidos, particularmente a presença de vários tipos de comportamentos desviantes (e.g., absentismo escolar; consumos de álcool e drogas) e até delinquentes e criminais (e.g., agressões físicas com colegas, professores, tráfico de droga, furtos, entrada em propriedades privadas) nesta localidade torna-se necessário apostar cada vez mais na prevenção deste tipo de comportamentos. Assim, é importante que os esforços de prevenção privilegiem este grupo de jovens que precocemente manifestam comportamentos antissociais, dado o seu maior risco para o desenvolvimento de futuras formas de inadaptação social (Fonseca, 2013). Sugere-se, deste modo, a imperativa necessidade de se continuar a apostar no desenvolvimento de programas de prevenção dos diferentes comportamentos de risco (e.g., condutas antissociais, consumos) associados à adolescência e à juventude, incidindo mais diretamente junto dos jovens, inseridos em meios urbanos e rurais, em de modo a estabelecer uma relação de confiança com ele. Importa igualmente atuar ao nível dos espaços escolares. Neste âmbito, a realização de programas de mediação escolar poderá contribuir para uma resolução mais eficaz e positiva dos conflitos em meio escolar, podendo constituir uma mais valia na prevenção do comportamento delinquente. É uma realidade que o espaço escolar é, não raras vezes, palco para a manifestação de diversos conflitos interpessoais (e.g., problemas de indisciplina, bullying, insucesso ou abandono escolar e violência entre pares), atendendo a que a grande maioria destas alterações comportamentais está sobretudo associada a dinâmicas familiares perversas, como sejam a exposição a violência doméstica, fracas relações parentais, desemprego, condições económicas desfavoráveis, álcool, tráfico e consumo de droga. Importa, pois, continuar a promover esforços interventivos e preventivos que incidam de forma particular na eliminação destas dinâmicas familiares e promoção dos vínculos parentais.

Por fim, importa destacar a necessidade de se privilegiar a adoção e implementação precoces de estratégias de prevenção do comportamento delinquente, de forma a evitar que este progrida para a idade adulta, tornando mais árduo o processo interventivo e remediativo.

Conflito de interesses | Conflict of interest: nenhum | none.

Fontes de financiamento | Funding sources: FCT - Fundação para a Ciência e a Tecnologia no âmbito do projeto PTDC/DIRDCP/28120/2017 | FCT - Fundação para a Ciência e Tecnologia [Portuguese national funding agency for science, research and technology] under the project PTDC / DIR-DCP / 28120/2017.

Contributos | Contributions: SC e ACM: Revisão da literatura; Recolha, inserção e tratamento dos dados; redação do manuscrito. LN: Contributo na redação do manuscrito; Tratamento e discussão dos dados; Revisão da redação final do manuscrito.

Declaração | Acknowledgments: Partes deste trabalho foram apresentadas em cumprimento parcial dos requisitos para o grau de mestre na Faculdade de Ciências Humanas e Sociais da Universidade Fernando Pessoa, Porto, Portugal | Portions of this work have been submitted in partial fulfillment of the requirements for a master degree, at Faculty of Humanities and Social Sciences, University of Fernando Pessoa, Porto, Portugal.

\section{Referências}

Almeida-Paiva, M. O., \& Lourenço, A. A. (2010). Comportamentos disruptivos e sucesso académico: A importância de variáveis psicológicas e de ambiente [Disruptive behavior and academic achievement: The importance of psychological and environment variables]. Revista Argentina de Ciencias del Comportamiento, 2(2), 18-31. Retrieved from https://revistas.unc.edu.ar/index.php/racc/article/view/5260/5423 
Amaral, I., Reis, B., Lopes, P., \& Quintas, C. (2017). Práticas e consumos dos jovens portugueses em ambientes digitais [Practices and consumption of Portuguese youth in digital environments]. Estudos em Comunicação, 201724), 107-131. https://doi.org/10.20287/ec.n24.a06

Barbosa-Ducharne, M., Cruz, O., Marinho, S., \& Grande, C. (2012). Estilos de vida adolescente: Exploração de rotinas diárias à semana e ao fim de semana [Teen lifestyles: Exploring daily routines at week and at weekends]. Revista Amazônica, 8(1), 149-172. Retrieved from https://sigarra.up.pt/fpceup/pt/pub_geral.show_file?pi_doc_id=22508

Batista, V., Carvalho, S., Pontes, T., \& Antunes, H. (2015). Consumo de droga entre adolescentes institucionalizados [Drug consumption between adolescents institutionalized] [Abstract]. Nascer e CrescerRevista de Pediatria do Centro Hospitalar do Porto, 24(Suppl. 2), S12. https://doi.org/10.25753/BirthGrowthMJ.v24.i0.9431

Bidarra, M. G. A., Vaz-Rebelo, M. P., Barreira, C. M. F., Alferes, V. A. R., \& Pereira, A. C. C. (2017). Autoconceito, hábitos de estudo, procrastinação e rendimento escolar: Que relação? [Self-concept, study habits, procrastination and school performance: What relationship?]. Revista de Estudios e Investigación en Psicología y Educcación, Extr.(1), 174-175. https://doi.org/10.17979/reipe.2017.0.01.2491

Born, M. (2005). Psicologia da delinquência [Psychology of Delinquency]. Lisboa: Climepsi Editores.

Braga, T., \& Gonçalves, R. J. A. C. (2013). Delinquência juvenil: Da caracterização à intervenção [Juvenile delinquency: From characterization to intervention]. Revista de Psicologia da Criança e do Adolescente, 4(1), 95-116. Retrieved from http://revistas.lis.ulusiada.pt/index.php/rpca/article/view/93/88

Braga, T., Gonçalves, L. C., Basto-Pereira, M., \& Maia, Â. (2017). Unraveling the link between maltreatment and juvenile antissocial behavior: A meta-analysis of prospective longitudinal studies. Aggression and Violent Behavior, 33, 37-50. https://doi.org/10.1016/j.avb.2017.01.006

Braga, T., Pechorro, P., Jesus, S. N., \& Gonçalves, R. A. (2018). Autoestima, narcisismo e dimensões de delinquência juvenil: Que relação? [Self-esteem, narcissism, and dimensions of juvenile delinquency: Clarifying the relation]. Análise Psicológica, 36(2), 145-157. https://doi.org/10.14417/ap.1361

Cardoso, L. R. D., \& Malbergier, A. (2014). A influência dos amigos no consumo de drogas entre adolescentes [Friend's influence on drug consumption among adolescents]. Estudos de Psicologia (Campinas), 31(1), 6573. https://doi.org/10.1590/0103-166X2014000100007

Carvalho, M. J. L. (2005). Jovens e delinquências: (Sobre)vivências na família [Young people and delinquencies: (About)family experiences]. Psicologia, 18(2), 129-158. Retrieved from http://www.scielo.mec.pt/pdf/psi/v18n2/v18n2a06.pdf

Carvalho, M. J. L. (2010). Do outro lado da cidade: Crianças, socialização e delinquência em bairros de realojamento [The other side of the city: Children, socialization and delinquency in public housing neighbourhoods] (Doctoral dissertation, Universidade Nova de Lisboa, Lisboa). Retrieved from http://run.unl.pt/handle/10362/6132

Carvalho, M. J. L., \& Duarte, V. M. (2013). Crianças, jovens e a cidade: Riscos, violências e delinquências em Portugal [Children, youth and the city: Risks, violence and delinquencies in Portugal]. Latitude, 72), 133-166. https://doi.org/10.28998/2179-5428.20130208

Chesney-Lind, M. (1997). The female offender: Girls, women, and crime. Thousand Oaks, CA: Sage Publications.

Cho, S., Wooldredge, J., \& Park, C. S. (2016). Lifestyles/routine activities and bullying among South Korean youths. Victims \& Offenders, 11(2), 285-314. https://doi.org/10.1080/15564886.2014.982235

Conde, R., \& Teixeira S. (2018). Delinquência juvenil em Portugal: Estudo qualitativo das histórias de vida de jovens reclusos [Juvenile delinquency in Portugal: Qualitative study of the life stories of young prisoners]. Revista Psicologia, Diversidade e Saúde, 71), 78-90. https://doi.org/10.17267/2317-3394rpds.v7i1.1844

Cohen, J. (1988). Statistical power analysis for the behavioral sciences (2nd ed.). New York: Lawrence Erlbaum Pub. 
Cunha, C., Soares, M., Veríssimo, L., \& Matos, R. (2015). Família, pares e delinquência juvenil: Análise de diferentes percursos de reincidência [Family, peers and juvenile delinquency: Analysis of different pathways to recidivism]. Revista de Psicología, 24(2), 1-18. https://doi.org/10.5354/0719-0581.2015.38134

Dalosto, M. M., \& Alencar, E. M. L. S. (2013). Manifestações e prevalência de bullying entre alunos com altas habilidades/superdotação [Bullying manifestations and prevalence among gifted students]. Revista Brasileira de Educação Especial, 19(3), 363-378. https://doi.org/10.1590/S1413-65382013000300005

Dias, I. (2016). Inquérito de vitimação [Victimization survey]. In R. L. Maia, L. M. Nunes, S. Caridade, A. I. Sani, R. Estrada, C. Nogueira, H. Fernandes, \& L. Afonso (Eds.), Dicionário: Crime, justiça e sociedade [Dictionary: Crime, justice and society] (pp. 269-270). Lisboa: Edições Sílabo.

Duarte, V. M. (2013). Discursos e percursos na delinquência juvenil feminina[Discourses and pathways in female juvenile delinquency]. Lisboa: Edições Húmus.

Duarte, V. M., \& Carvalho, M. J. L. (2012). (Entre) olhares sobre a delinquência juvenil no feminino [Looks on female delinquency]. Ex æquo, 2012(28), 31-44. Retrieved from https://repositorio.ismai.pt/bitstream/10400.24/196/1/3VD.pdf

Espírito-Santo, H., \& Daniel, F. (2015). Calcular e apresentar tamanhos do efeito em trabalhos científicos (1): As limitações do $p<0,05$ na análise de diferenças de médias de dois grupos [Calculating and reporting effect sizes on scientific papers (1): $\mathrm{p}<0.05$ limitations in the analysis of mean differences of two groups]. Revista $\begin{array}{lllllll}\text { Portuguesa de Investigação Comportamental } & e & \text { Social, } & 1 & \text { (1), 3-16. }\end{array}$ https://doi.org/10.7342/ismt.rpics.2015.1.1.14

Farrington, D. P., Gaffney, H., \& Ttofi, M. M. (2016). Systematic reviews of explanatory risk factors for violence, offending, and delinquency. Aggression and Violent Behavior, 33, 24-36. https://doi.org/10.1016/j.avb.2016.11.004

Fonseca, A. C. (2013). Consumo de drogas e comportamentos antissociais na adolescência: Que relação? [Substance use and antisocial behaviour across adolescence: Which relationship?]. Revista Portuguesa de Pedagogia, 471), 157-176. https://doi.org/10.14195/1647-8614_47-1_8

Forsyth, C. J., Dick, S. J., Chen, J., Biggar, R. W., Forsyth, Y. A., \& Burstein, K. (2018). Social psychological risk factors, delinquency and age of onset. Criminal Justice Studies, 31(2), 178-191. https://doi.org/10.1080/1478601X.2018.1435618

Garcia, J. (2006). Indisciplina, incivilidade e cidadania na escola [Indiscipline, incivility and citizenship at school]. Educação Temática Digital, 8(1), 121-130. https://doi.org/10.20396/etd.v8i1.1112

Gersão, E., \& Lisboa, M. (1994). The self-report delinquency study in Portugal. In J. Junger-Tas, G.-J. Terlouw, \& M. W. Klein (Eds.), Delinquent behaviour among young people in the western world: First results of the international self-report delinquency study (pp. 212-237). Amsterdam: Kugler Publications.

Gomes, H. M. S., \& Gouveia-Pereira, M. (2014). Funcionamento familiar e delinquência juvenil: A mediação do autocontrolo [Family functioning and juvenile delinquency: The mediation of self-control]. Análise Psicológica, 32(4), 439-451. https://doi.org/10.14417/ap.958

Gottfredson, M., \& Hirschi, T. (1990). A general theory of crime. Stanford, CA: Stanford University Press.

Hipwell, A. E., \& Loeber, R. (2006). Do we know which interventions are effective for disruptive and delinquent girls? Clinical Child and Family Psychology Review, 93-4), 221-255. https://doi.org/10.1007/s10567-0060012-2

Içli, T. G., \& Çoban, S. (2012). A study on the effects of family and deliquent peers on juvenile delinquency in Turkey. Advances in Applied Sociology, 2(1), 66-72. https://doi.org/10.4236/aasoci.2012.21009

Instituto Nacional de Estatística. (2017). Online database. Retrieved from https://www.ine.pt/xportal/xmain?xpid=INE\&xpgid=ine_base_dados\&contexto=bd\&selTab=tab2 [Acessed 10 May 2018]

Leão, L. G. R. (2010). O fenômeno bullying no ambiente escolar [The bullying phenomenon in the school environment]. Revista FACEVV, 4, 119-135. 
Malta, D. C., Mascarenhas, M. D. M., Porto, D. L., Duarte, E. A., Sardinha, L. M., Barreto, S. M., \& Morais Neto, O. L. (2011). Prevalence of alcohol and drug consumption among adolescents: Data analysis of the National Survey of School Health. Revista Brasileira de Epidemiologia, 14(Suppl. 1), 136-146. https://doi.org/10.1590/S1415-790X2011000500014

Marôco, J. (2010). Análise estatística com o PASW Statistics (ex-SPSS)[Statistical analysis with PASW Statistics (ex-SPSS)]. Pêro Pinheiro: ReportNumber.

Matos, R. (2008). Vidas raras de mulheres comuns: Percursos de vida, significações do crime e construção da identidade em jovens reclusas [Rare lives of ordinary women: Life paths, meanings of crime and identity building in young inmates]. Coimbra: Almedina.

Meldrum, R. C., Young, J. T. N., Hay, C., \& Flexon, J. L. (2012). Does self-control influence maternal attachment? A reciprocal effects analysis from early childhood through middle adolescence. Journal of Quantitative Criminology, 28(4), 673-699. https://doi.org/10.1007/s10940-012-9173-y

Mendes, S. M., \& Carvalho, S. (2010). Portugal. In J. Junger-Tas, I. H. Marshall, D. Enzmann, M. Killias, M. Steketee, \& B. Gruszczynska (Eds.), Juvenile delinquency in Europe and beyond: Results of the second international selfreport delinquency study (pp. 205-212). New York, NY: Springer. https://doi.org/10.1007/978-0-38795982-5

Negreiros, J. (2008). Delinquência juvenis - Trajectórias, intervenção e prevenção [Juvenile delinquencies Trajectories, interventions and prevention]. Porto: Livpsic, Legis Editora.

Negreiros, J. (2016). Delinquência [Deliquency]. In R. L. Maia, L. M. Nunes, S. Caridade, A. I. Sani, R. Estrada, C. Nogueira, H. Fernandes, \& L. Afonso (Coords.), Dicionário crime, justiça e sociedade [Crime, justice and society] (1st ed., pp. 140-150). Lisboa: Edições Sílabo.

Newman, K., Harrison, L., Dashiff, C., \& Davies, S. (2008). Relationships between parenting styles and risk behaviors in adolescent health: An integrative literature review. Revista Latino-Americana de Enfermagem, 16(1), 142-150. https://doi.org/10.1590/S0104-11692008000100022

Nunes, L. M., Beça, S., \& Dinis, M. A. P. (2017). Drug abuse and trafficking in universities: An emerging social phenomenon. In J. A. Jaworski (Ed.), Advances in sociology research (Vol. 23, pp. 179-192). New York, NY: Nova Science Publishers.

Nunes, L. M., Caridade, S., \& Sani, A. I. (2017). Incivilities and delinquency in schools: A social phenomenon in analysis. In J. A. Jaworski (Ed.), Advances in sociology research (Vol. 23, pp. 193-207). New York, NY: Nova Science Publishers.

Nunes, L. M., \& Trindade, J. (2015). Delinquência: Percursos criminais - Desenvolvimento, controle, espaço físico e desorganização social [Delinquency: Criminal routes - Development, control, physical space and social disorganization] (1st ed.). Porto Alegre: Livraria do Advogado.

Pauwels, L., \& Svensson, R. (2009). Adolescent lifestyle risk by gender and ethnic background: Findings from two urban samples. European Journal of Criminology, 6(1), 5-23. https://doi.org/10.1177/1477370808098102

Pechorro, P., Marôco, J., Vieira, R. X., Oliveira, J. P., \& Gonçalves, R. A. (2015). Delinquência juvenil: Um estudo comparativo de rapazes institucionalizados [Juvenile delinquency: A comparative study of institutionalized boys]. Revista de Psicologia da Criança e do Adolescente, 6(2), 59-75.

Pimenta, S., \& Nunes, L. M. (2017). A escola como espaço de violência e incivilidades: O meio, a arquitetura e a caraterização espacial [The school as a space of violence and incivilities: The environment, architecture and spatial characterization]. In L. M. Nunes, A I. Sani, R. L. Maia, S. Caridade, F. Viana, \& R. Estrada (Coords.), Crime e segurança nas cidades contemporâneas [Crime and security in contemporary cities] (121-138). Porto: Fronteira do Caos.

Pujazon-Zazik, M., \& Park, M. J. (2010). To tweet, or not to tweet: Gender differences and potential positive and negative health outcomes of adolescents' social internet use. American Journal of Men's Health, 4(1), 77-85. https://doi.org/10.1177/1557988309360819

Rosenthal, R. (1991). Meta-analytic procedures for social research (Revised). Newbury Park: Sage. 
Sani, A., Nunes, L. M., \& Caridade, S. (2016). Child exposure to domestic violence: The risk of drug abuse and dating violence. In M. Ortiz (Ed.), Domestic violence: Prevalence, risk factors and perspectives (pp. 69-82). USA: Nova Science Publishers, Inc.

Simões, S., Ferreira, J. J., Braga, S., \& Vicente, H. T. (2015). Bullying, vinculação e estilos educativos parentais em adolescentes [Bullying, attachment and parental rearing styles in adolescentes from the 3rd cycle of basic school]. Revista Portuguesa de Investigação Comportamental e Social, 1(1), 30-41. https://doi.org/10.7342/ismt.rpics.2015.1.1.8

Sintra, C. I. F., \& Formiga, N. S. (2012). Condutas desviantes e habilidades sociais em jovens portugueses toxicodependentes e não-toxicodependentes [Deviant behaviors and social skills in young Portuguese drug addicts and non-addicts]. Encontro: Revista de Psicologia, 15(23), 9-25.

Svensson, R., \& Pauwels, L. (2010). Is a risky lifestyle always "risky"? The interaction between individual propensity and lifestyle risk in adolescent offending: A test in two urban samples. Crime \& Delinquency, 56(4), 608-626. https://doi.org/10.1177/0011128708324290

Walters, G. D. (1990). The criminal lifestyle: Patterns of serious criminal conduct. Newbury Park, CA: Sage Publications. https://doi.org/10.4135/9781483325569

Walters, G. D. (2016). Proactive criminal thinking and deviant identity as mediators of the peer influence effect. Youth Violence and Juvenile Justice, 15(3), 281-298. https://doi.org/10.1177/1541204016636436

Walters, G. D. (2017). Mediating the relationship between parental control/support and offspring delinquency: Self-efficacy for a conventional lifestyle versus self-efficacy for deviance. Crime \& Delinquency, 64(5), 606624. https://doi.org/10.1177/0011128716686357 